\title{
¿Que hay de nuevo en Bolivia con Evo Morales?
}

\section{Juan Téllez Rodríguez}

\begin{abstract}
El vivir bien es subversivo al capitalismo neoliberal y globalizado, y está cargado de utopías esperanzadoras para trascender la oscuridad del instante vivido. En 2006 irrumpió el vivir bien con el arribo al poder del primer presidente indigena de Bolivia: Evo Morales. Siete años después de aquel acontecimiento en Tiwanaku (el 21 de enero, 2012) dirigimos la mirada hacia atrás para ver las huellas en el camino recorrido.
\end{abstract}

Mientras los Pueblos Indigenas proponen para el mundo el «Vivir Bien», el capitalismo se basa en el «Vivir Mejor».

Evo Morales, 2011

Mi tributo póstumo al P. Gregorio Iriarte que cultivó la entrega generosa en toda su vida y a Freddy Beltrán que soñó y donó su vida por una Patria liberada.

\section{LA HUELLA DE LA ESPERANZA}

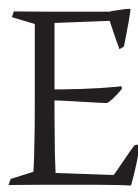
n enero de 2006 se subvierten en Bolivia dos flujos de coexistencia histórica: el flujo dominante del vivir mejor que viene desde el siglo XVI y la emergente cosmovisión comunitaria del vivir bien que viene con las culturas milenarias. A diferencia de otras experiencias del pasado, ahora llega al poder, con voz propia, el sujeto histórico del vivir bien: los movimientos sociales y las naciones indígena-campesinas. Esto no significa que las prácticas del vivir mejor hayan desaparecido, porque siguen presentes en formas variadas. Además, los sistemas de reciprocidad-complementariedad necesitan entrar más al centro del proceso. Tenemos, entonces, dos flujos:

a) Los agentes del paradigma dominante, que organizaron el territorio y formaron relaciones sociales de producción en el marco del colonialismo feudal y, más tarde, del imperialismo capitalista. Este proceso fue una acumulación frenética de la riqueza para vivir mejor a costa del Otro, ${ }^{2}$ de las comunidades indígenas, de los/ as trabajadoras de la periferia. En 1545 los españoles llegaron a Potosí para saquear la plata del Cerro Rico relocalizando comunidades de los Chichas, Yuras, Quechuas y 
otros, para proveer mano de obra barata a las minas, y usurparon las tierras comunitarias para suministrar alimentos. Para los conquistadores no fue suficiente la mano de obra indígena, y en 1557 trajeron, vía Puerto Madero (ahora Buenos Aires), hermanos/ as de África en categoría de esclavos, ${ }^{3}$ y expandieron con ellos el plusvalor absoluto en la extracción de los minerales. Este modus operandi atravesó las décadas del liberalismo oligárquico-feudal vinculado al imperialismo estadunidense en el siglo $\mathrm{xx}$, hasta llegar a las dos décadas de neoliberalismo (1985-2005), que para garantizar el saqueo desregularon las relaciones sociales de producción, privatizaron las empresas estatales y entregaron los recursos naturales a los inversionistas extranjeros en el proceso de acumular capital en escala global para que otros vivan mejor. El desarrollo capitalista generado en este flujo está presentado, por muchos, como un gran avance en la marcha de la humanidad, aunque los costos sociales y ambientales de este «progreso» están derivando en una crisis global.

b) La cosmovisión indígena es el flujo de prácticas comunitarias originaria-campesinas que en el cautiverio han resistido al colonialismo-imperialismo, cultivando estructuras productivas-reproductivas sustentadas en relaciones sociales de solidaridad, reciprocidadcomplementariedad entre los seres humanos y en armonía con la naturaleza. En 1781, la insurgencia de Bartolina Sisa y Tupac Katari irrumpió la historia reivindicando el territorio usurpado. En la guerra de independencia (1810-1825), los indígenas y afrobolivianos buscaron su libertad y Simón Bolívar les prometió abolir la esclavitud con la fundación de la República. En realidad, los criollos (terratenientes descendientes de europeos - el colonialismo interno) borraron ese acápite de la constitución bolivariana. ${ }^{4}$ La Tesis de Pulacayo fue una contribución majestuosa de los mineros que venían de las entrañas de las comunidades indígenas. Esa tesis fue la guía para el fin de la oligarquía imperialista con la revolución nacional de 1952. ${ }^{5}$ Más tarde, Domitila Chungara, minera-indígena, junto a otras mujeres, con la huelga de hambre en diciembre 1977, en articulación con la Central Obrera Boliviana, abrazó la utopía de la libertad hasta arrancarle a la dictadura militar la amnistía para los exilados y las elecciones generales. ${ }^{6}$ La Marcha por el Territorio y la Dignidad de los pueblos

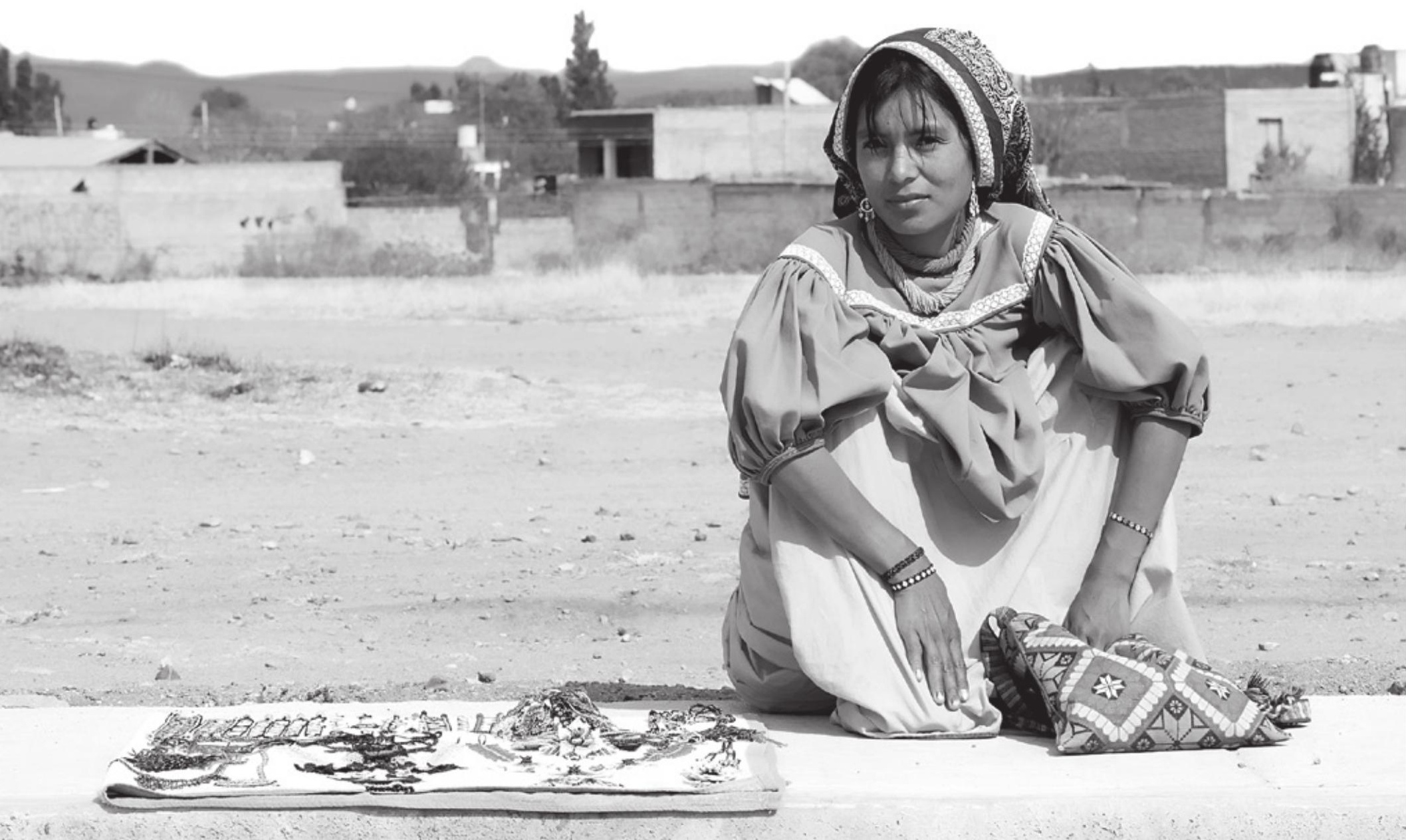


indígenas de las tierras bajas, en agosto de 1990, la Guerra del Agua en abril de 2000 y la Guerra del Gas en octubre de 2003 pertenecen a este ovillo de liberación en el que está cultivado el carácter subversivo del vivir bien. Con esta fuerza histórica de los pobres, llena de esperanza y utopía del vivir bien, llega al poder Evo Morales. En el gráfico 1 se refleja esta convergencia de flujos históricos.

GRÁFICO 1

Flujo de paradigmas históricos.

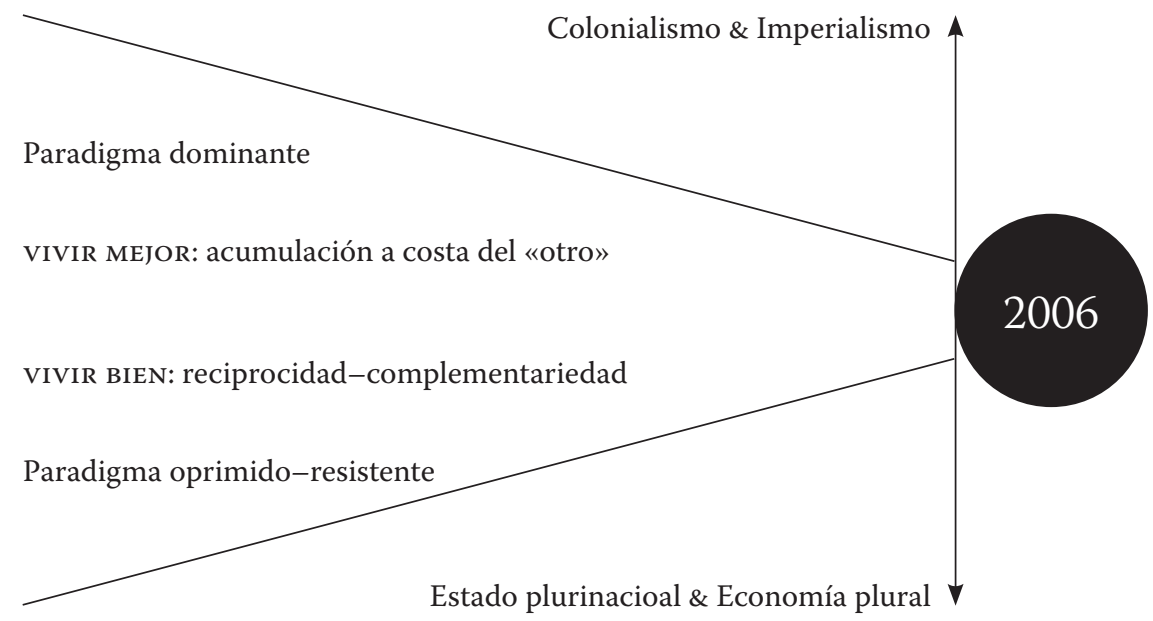

Mercado-Estado-Socio Centrismo vs Vida-céntrica

Fuente: elaboración propia.

\section{LAS HUELLAS DEL CAMBIO}

Tiwanaku, 21 de enero de 2006. Nadie olvidará aquella mañana de sol radiante y de toda la energía de los Andes en la posición de Evo Morales. «Mis ojos no pueden creer lo que ven» dijo, con tono bíblico, el P. Javier Albó, investigador reconocido en Bolivia. «Esto había que vivirlo», dijo emocionado Fernando Mayorga, el sociólogo que analiza la sociedad boliviana desde tantos ángulos, en la Plaza Murillo al ver investido a Evo. Para mí, parafraseando a la inversa al dramaturgo de la ilustración española, Calderón de la Barca, no todo en la vida es sueño, ni todos los sueños, sólo sueños son. Ésta es la primera huella histórica de Evo: "Ya» estaba entre nosotros la descolonización, pero «todavía no» plenamente, hay mucho camino que recorrer y construir.
Un regalo en el día de los trabajadores. El 1 de mayo de 2006, los hidrocarburos son arrancados de manos de las transnacionales petroleras que fueran concedidos por los gobiernos neoliberales a precio de «gallina muerta». Para algunos críticos desubicados en el desarrollo de Bolivia, esta medida es un regreso al Estado-centrismo keynesiano. Desde la perspectiva del proceso de cambio, es una medida de doble propósito: a) cambiar la matriz productiva primario exportadora hacia la industrialización de los recursos naturales, y b) apropiarse de la generación de excedentes de sectores estratégicos para distribuirlos en el desarrollo social-comunitario, en la economía plural y el fortalecimiento del proceso autonómico, para la transformación hacia el socialismo. ${ }^{7}$ Poco tiene que ver ésta medida con el nuevo desarrollismo de Breisser-Pereira, porque busca a largo plazo el cambio estructural socialista y no sólo una reforma al modelo capitalista.

Nueva configuración del territorio. Bolivia ya no es una República, es un Estado plurinacional, social, comunitario y autonómico. La Constitución Política del Estado (CPE) se elabora en 2008 con la participación de partidos políticos y la representación directa de las bases sociales que viven en el territorio boliviano. La CPE es el reco- 
nocimiento de la diversidad étnica y de los usos y costumbres en las comunidades milenarias; es la superación al posmodernismo que reduce los hechos a la microfísica del poder hundiendo las particularidades en un nihilismo absurdo de la historia. Hay lugar en la CPE para la individualidad, diversidad, complementariedad y reciprocidad en el marco del vivir bien.

Plan Nacional de Desarrollo (PND). Es la perspectiva programática del vivir bien para desmontar el «doble colonialismo» y la dependencia al capitalismo globalizado. La construcción de las «cuatro Bolivias» - soberana, digna, democrática y productivaresume el espíritu de la nueva Bolivia para el presente y el futuro. En el PND el cambio estructural tiene dos pilares estratégicos: a) la matriz social comunitaria: enfocada en la generación de activos sociales (salud, educación, infraestructura), donde el Estado es protagonista, y la creación de condiciones sociales (justicia, equidad, diversidad cultural) donde el Estado es promotor-facilitador, y b) la matriz productiva diversificada, de transformación y comunitaria, con el Estado protagonista (dueño) de las inversiones en sectores económicos estratégicos para generar excedentes y redistribuir a favor del desarrollo social, la construcción de la economía plural y resolver las asimetrías socioeconómicas.

Economía Plural. La Economía Plural (EP) es el reflejo del Estado Plurinacional y reconoce la diversidad de modos de organizar la producción que han coexistido de manera dialéctica y envueltas en relaciones de poder. El PND apuesta a fortalecer la economía comunitaria para cambiar la asimetría en la generación-distribución de la riqueza. En esta economía están incubadas las relaciones de reciprocidad-complementariedad para vivir bien. La gama de desarrollistas, desde clásicos hasta marxistas radicales, sólo ven deficiencia en este modo de producción, y es visto como un obstáculo para la modernización y el crecimiento económico. Se trata de una mirada necia de la modernización, porque no la aprecian en su forma milenaria-humanizadora. En la EP también están la economía asociada y la economía privada para la generación de ingresos y empleo como parte de la construcción del vivir bien. La economía estatal en la EP tiene dos dimensiones: una ligada al capitalismo de Estado y otra al socialismo empresarial.

Una huella soberana. Antes de 2006, la embajada de Estados Unidos tenía más autoridad sobre el destino de Bolivia que los bolivianos/as o los ministros de Estado. El Fondo Monetario Internacional (FMI) y el Banco Mundial (BM) tenían en sus manos la carta de condicionalidad con la que sometían a Bolivia a los intereses de las transnacionales. En 2006 se desalojó a los estadounidenses uniformados junto con la Administración Federal Antidrogas (DEA, por sus siglas en inglés), y se acabó con las operaciones de la embajada estadounidense que seguía operando mediante organizaciones no gubernamentales (ONG) y agentes encubiertos para desestabilizar al gobierno de Evo. Desde 2006, se cambiaron las relaciones diplomá- ticas para cooperar con Cuba, Venezuela, Irán, China, Corea y otros países. También cambiaron las formas de colaboración con la cooperación internacional, para asegurar que ellos se adecuen al proyecto de Bolivia y al revés. ${ }^{8}$ También se desarrollaron nuevas formas de integrarse a la región a través de la Alianza Bolivariana para los Pueblos de Nuestra América (Alba), la Unión de Naciones Suramericanas (Unasur) y, últimamente, el Mercado Común del Sur (Mercosur).

\section{Otras huellas}

Huellas de diferencia. Hay un crecimiento económico constante del producto interno bruto (PIB) de $4 \%$ en 2006 a 6.1\% en 2012, con una mejor distribución de los excedentes a través de programas sociales (bonos: dignidad, Juancito Pinto, Juan Azurduy, habilidades diferentes y las transferencias a los gobiernos autónomos), con iniciativas productivas y para implementar la Ley de Revolución Productiva Comunitaria Agropecuaria. El Programa de Naciones Unidas para el Desarrollo (PNUD) de Bolivia felicitó a Evo Morales por el crecimiento económico con distribución equitativa y la reducción de la pobreza extrema a $14 \%$, y el cambio del GINI de 0.57 en 2000 a 0.43 en 2011. Desde 2006, las personas de comunidades indígenas son más visibles en los órganos legislativo y ejecutivo y en el sistema de educación superior.

Huellas de colores de la primera gestión (2006-2009)9 y la segunda (2010-2012). La primera dio más luces hacia el socialismo y la realización del vivir bien, con la nacionalización de hidrocarburos, telecomunicaciones, transporte aéreo, bonos. Dieron signos de nuevos tiempos de soberanía y dignidad, abriendo espacios de participación de indígenas y mujeres en los órganos del Estado. También se elaboraron los nuevos paradigmas en la CPE y el PND. En la segunda, si bien se elaboraron los marcos jurídicos - la Ley Marco de Autonomías, la Ley contra el Racismo y Toda Forma de Discriminación, 
la Ley de la Revolución Productiva Comunitaria Agropecuaria - terminaron por estancarse en manos de lentas burocracias y son el talón de Aquiles. Tiene más fuerza el pragmatismo. Así, por ejemplo, el Ministerio de Planificación del Desarrollo, que iluminó el camino con el PND, fue desmantelado y hoy se dedica a conseguir fondos financieros en el exterior.

Huellas de otra lógica. El gasolinazo en diciembre de 2010 (subida de precios de hidrocarburos) fue un desacierto de estilo neoliberal, con impactos estructurales desprevenidos, lanzado aprovechando el feriado de navidad. Esto puso a Bolivia en llamas hasta hacer retroceder la medida. También la intención de construir la carretera por el Territorio Indígena y Parque Nacional Isiboro-Secure (TIPNIS) - por presión de los productores de la hoja de coca, base social de Evo, y el compromiso con Brasil para contar con la carretera transoceánica- llevó al gobierno a la represión torpe contra los indígenas que marchaban hacia La Paz, el 26 septiembre de 2011, para resistir el proyecto desarrollista y los impactos en la diversidad biológica y social. Lo más repugnante es la constante corrupción desde adentro del Movimiento al Socialismo (MAS) y del gobierno. Uno de los hombres de mayor nivel en el MAS, Santos Ramírez, fue sorprendido con un soborno de 80 millones de dólares. El ex jefe de la policía nacional, René Sanabria, fue capturado en Panamá con 144 kilos de cocaína. ${ }^{10} \mathrm{En}$ noviembre de 2012, una red de extorsionadores operaba desde los Ministerios de Gobierno y de la Presidencia. ${ }^{11}$

\section{Notas}

1 Juan Téllez es docente adjunto de la Universidad de Saint Mary's en Canadá y es estudiante del Doctorado en Estudios del Desarrollo de la Universidad Autónoma de Zacatecas. Fue asesor del Ministerio de Planificación del Desarrollo del Estado Plurinacional de Bolivia, 2009-2010.

2 Enrique Dussel, America Latina: Dependencia y Liberación, Buenos Aires, Fernando García Gambeiro, 1973.

3 Juan Téllez, El desarrollo del pueblo afrodescendiente en Bolivia. Ha sido un largo camino desde 1557 hasta nuestros dias...!, La Paz, Bolivia, Centro Regional para la Salvaguardia del Patrimonio Cultural Inmaterial de América Latina, CRESPIAL, Ministerio de Cultura de Bolivia, 2011.

4 James M. Malloy, Bolivia: la Revolución Inconclusa, El Alto, La Paz, CEREs, 1989.

5 Benjamin Dangl, El Precio del Fuego, La Paz, Plural, 2009.

6 Domitila Chungara y Moema Viezzer, Let me speak!: Testi-
Aunque es claro que Evo Morales no está involucrado ni impulsa los actos de corrupción, ésta existe.

\section{A MANERA DE CIERRE}

La plurinacionalidad del Estado boliviano está en proceso. A pesar de las huellas sombreadas de otra lógica, el actual proceso de cambio tiene perspectiva, porque es el triunfo del flujo de los movimientos sociales del vivir bien. Además, por lo menos seis años más, Evo Morales es la garantía de la continuidad de este proceso por sus cualidades de liderazgo. ${ }^{12}$

Hay una corriente que sostiene que Evo Morales es la cara indígena de fracaso del desarrollismo o que encarna una faceta indígena de la decadencia del capitalismo en Bolivia. A pesar de que algunos momentos parecen borrosos en el camino hacia el vivir bien, aunque a veces se hace difusa la utopía y la desesperanza se agranda porque hay tendencias en el gobierno de tinte Estado-céntrico y mercado-céntrico de corte capitalista, y éstos podrían desviar el proceso de cambio hacia horizontes del nuevo desarrollismo o hacia peores puertos, no hay duda de que estamos en el flujo histórico descolonizador, y con seguridad los movimientos sociales rectificarán la dirección de este proceso hacia el vivir bien. La construcción del Estado Plurinacional y de Economía Plural superarán estas tendencias para realizar, históricamente, las cosmovisiones esperanzadoras articuladas al vivir bien.

mony of Domitila, a Woman of the Bolivian Mines, New York, Montly Review Press, 1978.

7 Ministerio de Planificación del Desarrollo, Plan Nacional de Desarrollo, La Paz, Gaceta Oficial de Bolivia, 2009.

8 Hugo Moldiz, Bolivia en los Tiempos del Evo. Claves para entender el proceso boliviano, México, Ocean Sur Press, 2008.

9 Tanja Ernst y Schumalz (editores), El primer gobierno de Evo Morales: un balance retrospectivo, La Paz, Plural Editores, 2012.

10 Consultado noviembre 30 de 2012 en 〈http://online.wsj.com/ article/sB10001424053111903703604576589390650678946. html..

11 Consultado en diciembre 7 de 2012 en 〈http://www.elpotosi. net/2012/1201/14.php».

12 Fernando Mayorga, Dilemas. Ensayos sobre democracia intercultural y Estado Plurinacional, La Paz, Bolivia, CESU-PLURAL, 2011. 